Article

\title{
Lactobacillus acidophilus DDS-1 and Bifidobacterium lactis UABla-12 Improve Abdominal Pain Severity and Symptomology in Irritable Bowel Syndrome: Randomized Controlled Trial
}

\author{
Christopher J. Martoni ${ }^{1}(\mathbb{D})$, Shalini Srivastava ${ }^{2}(D)$ and Gregory J. Leyer ${ }^{1, *(D)}$ \\ 1 UAS Laboratories LLC, 4375 Duraform Lane, Windsor, WI 53598, USA; cmartoni@uaslabs.com \\ 2 Vedic Lifesciences, 203 Morya Landmark1, New Link Road, Andheri W, Mumbai 400053, India; \\ shalini.s@vediclifesciences.com \\ * Correspondence: gleyer@uaslabs.com
}

Received: 2 January 2020; Accepted: 28 January 2020; Published: 30 January 2020

\begin{abstract}
This randomized, double-blind, placebo-controlled, multi-center study investigated the clinical efficacy of two probiotic strains on abdominal pain severity and symptomology in irritable bowel syndrome (IBS). Three hundred and thirty adults, aged 18 to 70 years, with IBS according to Rome IV criteria were allocated (1:1:1) to receive placebo, Lactobacillus acidophilus DDS-1 $\left(1 \times 10^{10}\right.$ CFU/day) or Bifidobacterium animalis subsp. lactis UABla-12 $\left(1 \times 10^{10} \mathrm{CFU} /\right.$ day $)$ over six weeks. The primary outcome was the change in Abdominal Pain Severity - Numeric Rating Scale (APS-NRS). Over the intervention period, APS-NRS was significantly improved in both probiotic groups vs. placebo in absolute terms (DDS-1: $-2.59 \pm 2.07, p=0.001$; UABla-12: $-1.56 \pm 1.83, p=0.001$ ) and in percentage of significant responders (DDS-1: 52.3\%, $p<0.001)$; UABla-12 (28.2\%, $p=0.031$ ). Significant amelioration vs. placebo was observed in IBS Symptom Severity Scale (IBS-SSS) scores for L. acidophilus DDS-1 $(-133.4 \pm 95.19, p<0.001)$ and B. lactis UABla-12 $(-104.5 \pm 96.08, p<0.001)$ groups, including sub-scores related to abdominal pain, abdominal distension, bowel habits and quality of life. Additionally, a significant normalization was observed in stool consistency in both probiotic groups over time and as compared to placebo. In conclusion, L. acidophilus DDS-1 and B. lactis UABla-12 improved abdominal pain and symptom severity scores with a corresponding normalization of bowel habits in adults with IBS.
\end{abstract}

Keywords: Randomized controlled trial; irritable bowel syndrome; probiotic; abdominal pain; bowel habits; microbiome

\section{Introduction}

Irritable bowel syndrome (IBS), a functional disorder of the gastrointestinal tract, is characterized by chronic or recurrent abdominal pain and altered bowel habits [1]. Mechanisms include visceral hypersensitivity, altered bowel motility, abnormal epithelial barrier or intestinal permeability, immune activation, neurotransmitter imbalance, infection and dysbiosis of the microbiota [2,3]. Diagnosis of IBS is based on Rome IV criteria, a symptom-based classification system [4]. Current estimates report a worldwide prevalence of approximately $12 \%$, making IBS the most common functional gastrointestinal disorder [4]. Clinical management is challenging due to the heterogeneity of symptoms, and no validated treatment algorithm exists [5].

Systematic reviews have demonstrated a limited but significant effect of probiotics over placebo on IBS symptoms [6-9]. Probiotics are defined as live microorganisms that, when administered in adequate amounts, confer a health benefit to the host [10]. A recent meta-analysis assessed 37 randomized 
controlled trials with a dichotomous outcome enrolling a total of 4403 participants [7,11]. Probiotics were deemed superior to placebo regarding global symptoms, albeit with a weak recommendation and low strength of evidence [11]. Significant heterogeneity between studies and frequent limitations in study quality, design and power have prevented an optimal probiotic strategy for individuals with IBS. As such, more well powered randomized controlled trials are warranted.

The present study is unique in its assessment of single-strain Lactobacillus (acidophilus DDS-1) and Bifidobacterium (animalis subsp. lactis UABla-12) probiotic arms simultaneously in a randomized controlled trial. L. acidophilus DDS-1, alone or in combination with B. lactis UABla-12, has previously been shown to normalize bowel habits in functional constipation [12], provide abdominal symptom relief in lactose intolerance [13] and support immune specific outcomes [14,15] in randomized controlled trials. Mechanistically, L. acidophilus DDS-1 has exhibited immunomodulatory capacity in vitro [16], while both modulating the microbiota and downregulating the inflammatory profile in pre-clinical models $[17,18]$. Additionally, L. acidophilus DDS-1 in combination with B. lactis UABla-12 has demonstrated an immune regulatory role in support of immune specific outcomes clinically [15]. Further, a probiotic blend including both L. acidophilus DDS-1 and B. lactis UABla-12 was previously shown in a pilot trial to improve symptomology in participants with IBS [19]. The above pre-clinical and clinical evidence provided a rationale for a well-powered randomized controlled trial in IBS, while individually assessing L. acidophilus DDS-1 and B. lactis UABla-12.

The current study focused on abdominal pain as the primary outcome as it is a defining characteristic of IBS and a driver of healthcare resource utilization [20]. Unlike most symptoms, such as altered bowel habits or bloating, abdominal pain has been shown to independently drive health-related quality of life decline in IBS and is the principal driver of patient-reported symptom severity [21,22].

Therefore, the current randomized, double-blind, placebo-controlled, parallel-arm, multi-center study was conducted to explore the efficacy and tolerability of L. acidophilus DDS-1 and B. lactis UABla-12, simultaneously, with respect to abdominal pain severity, IBS related symptomology and bowel habits in adults with IBS.

\section{Subjects and Methods}

\subsection{Study Population}

Healthy adults, aged 18 to 70 years, meeting Rome IV diagnostic criteria for IBS were recruited from outpatient settings of 12 clinics specializing in gastroenterology practice in Greater Mumbai, India. As per Rome IV, included participants had recurrent abdominal pain for the last 3 months with symptom onset at least 6 months before diagnosis, and at least two of the following three symptoms: pain related to defecation or change in stool frequency or stool form. Additionally, participants had an average maximal abdominal pain severity of $\geq 4$ on the 11-point Abdominal Pain Severity -Numeric Rating Scale (APS-NRS) at screening and during the placebo run-in period as well as fasting blood glucose and hemoglobin levels at screening of $\leq 110 \mathrm{mg} / \mathrm{dl}$ and $\geq 10 \mathrm{~g} / \mathrm{dl}$, respectively. Participants with organic disease, a history of surgical resection of the stomach, small or large intestine, a history of inflammatory bowel disease, complications from infectious enteritis, hyperthyroidism or hypothyroidism, a history of diet-based intolerance, drug or alcohol abuse within the past 6 months, a history of malignant tumors, severe depression or anxiety disorder, or uncontrolled hypertension were excluded. Additional exclusion criteria included individuals with unstable medical conditions, type I or II diabetes, a history of cancer or abdominal surgery, immunocompromised conditions, or an active eating disorder. Smokers, defined as $\geq 1$ cigarette a day and individuals consuming $>2$ standard alcoholic drinks daily over the prior 3 months were excluded. The use of probiotic or fiber supplements, probiotic or fiber enriched foods, and IBS specific treatments were prohibited within 4 weeks of screening. Laxative medications or other agents affecting gastrointestinal motility were prohibited within 2 weeks of screening. All were prohibited during the trial. Lastly, individuals with an 
allergy or sensitivity to the study products and females who were pregnant, intended to get pregnant or breastfeeding were excluded.

\subsection{Study Design}

The study was conducted in accordance with the ethical principles that have their origins in the Declaration of Helsinki and its subsequent amendments. Study conduct was in full accordance with the study protocol and all applicable laws and regulations, including but not limited to current International Conference on Harmonisation - Good Clinical Practices, Schedule Y and the Indian Council of Medical Research Ethical Guidelines for Biomedical Research on Human Participants. The study was approved and monitored by an Independent Ethics Committee (Approval No: VED/P-01/03/FEB/2018; Address: ACEAS, Ahmedabad, Gujarat, India). The trial was registered on clinicaltrials.gov under study number NCT03482765 and was conducted according to the CONSORT 2010 Statement.

The study design was prospective, randomized, double-blind, placebo-controlled, multi-center and parallel-arm, including two active arms and one placebo arm. Participants visited the clinics at screening (day -18 \pm 2 ), and if applicable, randomization (day 0), day $21( \pm 2)$ and day $42( \pm 2)$ of the six-week intervention period. At screening, the study design was explained to participants in their local language following which written, signed and dated informed consent was obtained. Participants were assessed for all inclusion/exclusion criteria and medical history, concomitant medication, demographic details and vitals were recorded. Participants meeting screening criteria were dispensed with placebo product for a 14-day run-in period.

At randomization, APS-NRS over the placebo run-in period was reconciled and the average was considered as the baseline score. A decrease of more than 25\% in APS-NRS was classified as a placebo response and the participant was excluded from the study. Participants meeting the above criteria and exhibiting at least $80 \%$ of run-in product compliance were randomized. Participants were randomized at the ratio of 1:1:1 to receive either one of the investigational products (DDS-1 or UABla-12) or placebo based on the randomization schedule. Block randomization (block size: 6) was performed using Stats Direct software (version 3.2), generating distinct alphanumeric codes. Sites dispensed study products in sequential order of participant qualification. The randomization codes were concealed from investigators, as well as site and study teams involved in study conduct and evaluation, with blinding codes secured in tamper-evident sealed envelopes.

\subsection{Study Product}

The probiotic study products consisted of either L. acidophilus DDS ${ }^{\circledR}-1$ or B. animalis subsp. lactis UABla-12 ${ }^{\mathrm{TM}}$. Placebo and probiotic capsules (size " 1 " hypromellose) were prepared in compliance with standard operating and quality control procedures at UAS Laboratories LLC (Wausau, WI). L. acidophilus DDS-1 or B. lactis UABla-12 capsules, which each contained a potency of not less than (NLT) $1 \times 10^{10} \mathrm{CFU} /$ capsule, were formulated with lyophilized probiotic and microcrystalline cellulose. Placebo capsules were formulated with microcrystalline cellulose. All capsules contained minimal but identical quantities of magnesium stearate and silica as flow aids. Both finished formulations were non-allergenic. Probiotic and placebo capsules were identical in mass, taste and appearance and bottled in identical sealed $64 \mathrm{~mL}$ CSP bottles (CSP Technologies, Auburn, Alabama). Probiotic and placebo capsules were confirmed to meet all quality specifications, which included potency and bacterial culture purity, microbiological analyses, color, appearance, weight specification/variation and disintegration both at the time of manufacture and at the end of the clinical study period. Participants took the allotted study product orally, once daily before a meal, with a glass of water over the entire 42-day intervention period.

\subsection{Outcome Assessments}

Primary and secondary outcome assessments at baseline, midpoint and the end of study visits included abdominal pain severity, IBS symptom severity, stool form and frequency, IBS related quality 
of life and perceived stress. Additionally, a clinical examination was conducted, study product was dispensed, and study product compliance and concomitant medication usage was recorded. Mebeverine $\mathrm{HCl}(135 \mathrm{mg}$ ) as rescue medication to be taken in the case of severe pain was provided to the study participants for the length of the study.

The primary outcome was the change in abdominal pain severity over the intervention period. Abdominal pain severity was assessed via the 11-point APS-NRS [21], with 10 representing the most severe pain and 0 representing no pain. Post-screening APS-NRS scores were submitted using a digital diary on a weekly basis. An average of the prior 3 weeks' score was considered as the score for the corresponding visit.

IBS symptom severity was assessed via the IBS Symptom Severity Scale (IBS-SSS) [23]. Scores on the IBS-SSS range from 0 to 500, with 5 individual domains ( 0 to 100 ) related to abdominal pain severity, frequency of abdominal pain, severity of abdominal distention, dissatisfaction with bowel habits, and interference with quality of life. A decrease of 95 points has been associated with a clinically meaningful improvement [21].

Stool consistency was assessed via the Bristol Stool Scale (BSS), a validated ordinal scale of stool types [24] ranging from 1 through 7, with types 1-2 and 6-7, in conjunction with other symptoms, indicative of constipation and diarrhea, respectively. Types 3-5 are generally considered to be the most normal stool form and are the modal stool forms in cross-sectional surveys of healthy adults.

IBS-related quality of life (QoL) was assessed via the IBS-QoL, a 34-item questionnaire with each item rated on a 5-point scale (range: 34-170), with increasing scores indicating deteriorating quality of life [25]. Mental stress was assessed using the Perceived Stress Scale (PSS), a validated psychological instrument measuring the perception of stress and comprising negative and positive domains [26].

\subsection{Statistical Analysis}

The sample size calculation was based on the primary outcome, abdominal pain severity. A reduction in APS-NRS of at least 15\%, in comparison to placebo, was considered as a relevant treatment effect. Standard deviation (of the primary outcome) was estimated at $34 \%$. A placebo effect was estimated at 20-25\%. Assuming $80 \%$ study power and a two-sided statistical significance level of $5 \%$, sample size was estimated to be approximately 82 participants per group (15\% relative change compared to placebo). With an estimated drop-out rate of $15-20 \%$ after randomization, in this three-arm study, no less than 300 randomized participants were planned to be recruited at a ratio of 1:1:1, assuming a $15 \%$ relative change compared to placebo.

The primary and secondary objectives were assessed on the intention-to-treat (ITT) population. Descriptive statistics are presented as mean (standard deviation (SD)) or median (interquartile range (IQR)) for continuous variables or as a percentage for qualitative variables. Data normality was assessed using the Shapiro-Wilk test. Differences between groups for baseline characteristics were analyzed using a one-way ANOVA, a non-parametric Kruskal-Wallis test for continuous variables, or a Pearson Chi Square test for categorical variables. IBS-specific outcomes over the intervention period were determined to be sufficiently non-normally distributed. Therefore, differences between groups in IBS specific outcomes were assessed via a non-parametric Kruskal-Wallis test, followed by a Mann-Whitney $U$ to assess differences between individual treatment arms. Between group comparisons for categorical values over the intervention period, including responder rate and stool consistency profile, were assessed via a Pearson Chi Square test. Intragroup change in stool type was assessed via a McNemar test. Data processing, tabulation of descriptive statistics, and calculation of inferential statistics were performed using Statistical Package for the Social Sciences (SPSS, IBM ${ }^{\circledR}$ ) or Python 3.0 (Armonk, New York, USA). 


\section{Results}

\subsection{Study Parameters}

A total of 392 potential participants were screened and 336 subjects were randomized in the study. Screening and enrollment occurred continuously from April 2018 through March 2019. Three hundred and thirty subjects were randomized as part of the ITT population at a ratio of 1:1:1 with 109 subjects in the placebo group, 111 subjects in the DDS-1 group and 110 subjects in the UABla-12 group (Figure 1). Six subjects were incorrectly enrolled without meeting entry criteria and were excluded. Eleven subjects were lost to follow-up and one subject withdrew from the study at their request. Three hundred and eighteen subjects completed the study per protocol, with 106, 107 and 105 subjects in the placebo, DDS-1 and UABla-12 groups, respectively.

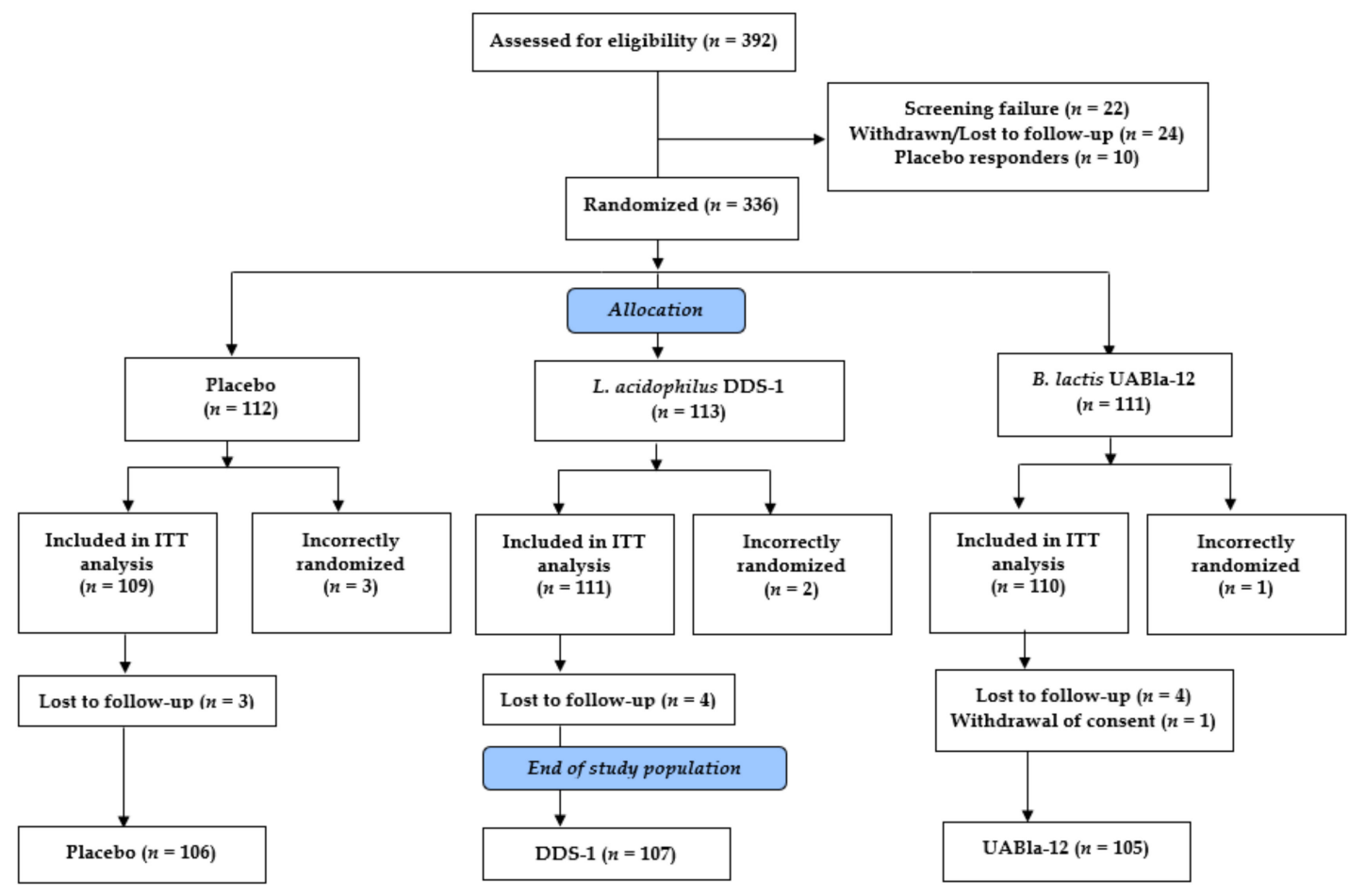

Figure 1. Participant flow chart. ITT: intention-to-treat.

\subsection{Baseline Characteristics of Subjects}

The baseline characteristics of the ITT population were evaluated and are presented in Table 1. The three groups were homogeneous in terms of demographic and clinical characteristics, including gender, body mass index, heart rate and blood pressure $(p>0.05)$. The mean age of subjects was higher in the B. lactis UABla-12 group (41.6 \pm 11.1 years) as compared to the placebo group (37.6 \pm 10.1 years). The ITT population comprised a nearly even distribution of females (46.6-52.3\%) and males in all three groups. The participants were deemed to have IBS per Rome IV criteria, with a mean duration of 20.8 months, 20.9 months and 22.1 months in the placebo, L. acidophilus DDS-1 and B. lactis UABla-12 groups, respectively. Subjects in all three groups presented similar baseline scores $(p>0.05)$ related to anxiety (generalized anxiety questionnaire) and overall health (participant health questionnaire). No smokers were enrolled in the study. Additionally, alcohol consumption profiles were similar between groups ( $p$ $>0.05$ ), with the vast majority of subjects not consuming alcohol. Dietary preferences were similar between groups with available evidence indicating a 2.1:1 ratio of non-vegetarians to vegetarians. All subjects enrolled in the trial were literate and living in urban areas. 


\subsection{Abdominal Pain Severity}

The groups were similar at baseline with mean APS-NRS scores of $6.94 \pm 1.02,7.03 \pm 0.99$ and 6.84 \pm 1.04 for the placebo, L. acidophilus DDS-1 and B. lactis UABla-12 groups, respectively. Significant between-group differences were observed in the change in abdominal pain severity score (primary outcome) over the intervention period for both probiotic groups when compared to placebo (Table 2). At day 42, APS-NRS showed a greater reduction in L. acidophilus DDS-1 $(-2.59 \pm 2.07)$ and B. lactis UABla-12 $(-1.56 \pm 1.83)$ groups, as compared to placebo $(-0.85 \pm 1.45)(p=0.001)$. L. acidophilus DDS-1 similarly showed a significant reduction at day $21(-1.24 \pm 1.10)$ as compared to placebo $(-0.71 \pm 0.92)$ $(p<0.001)$. Individuals considered to be significant responders to intervention, as defined by a greater than $30 \%$ reduction in abdominal pain severity, were significantly more numerous in L. acidophilus DDS-1 $(52.3 \%, p<0.001)$ and B. lactis UABla-12 $(28.2 \%, p=0.031)$ groups as compared to placebo $(15.6 \%)$ (Figure 2).

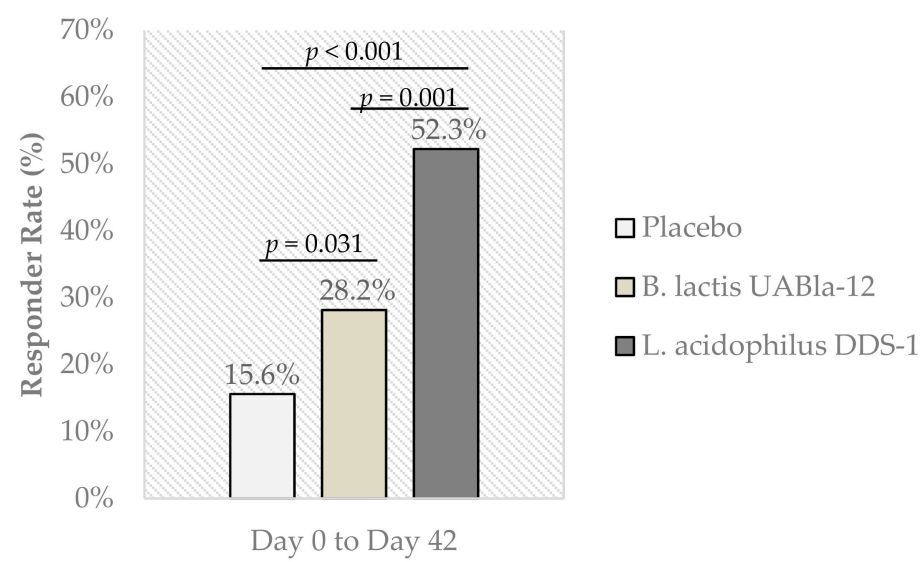

Figure 2. Percentage of significant responders, as defined by a decrease of $30 \%$ or more in the Abdominal Pain Severity - Numeric Rating Scale (APS-NRS) (primary outcome) from day 0 to day 42. Responder rate based on all subjects enrolled in ITT population. Between group comparison assessed by Pearson Chi Square test.

\subsection{IBS Symptomology}

IBS-SSS scores were similar at baseline with mean scores of $298.07 \pm 55.68,310.90 \pm 52.47$ and $305.45 \pm 48.82$ for placebo, L. acidophilus DDS-1 and B. lactis UABla-12 groups, respectively (Table 3). Over the intervention period, subjects receiving L. acidophilus DDS-1 capsules reported significant reductions as compared to placebo in IBS-SSS $(-133.4 \pm 95.19, p<0.001)$ with domain-specific scores related to abdominal pain severity $(-32.94 \pm 21.78, p<0.001)$, abdominal pain duration $(-28.44 \pm 24.80$, $p<0.001)$, abdominal distension $(-27.20 \pm 25.15, p<0.001)$, bowel habits $(-22.89 \pm 22.46, p<0.001)$ and quality of life $(-21.88 \pm 22.27, p<0.001)$. Significant differences between L. acidophilus DDS-1 and placebo were also observed after 21 days for IBS-SSS total score $(-56.06 \pm 57.64, p<0.001)$ and domains related to abdominal pain severity $(-15.50 \pm 15.23, p=0.001)$, abdominal pain duration $(-13.49 \pm 21.79$, $p=0.002)$, bowel habits $(-8.90 \pm 13.82, p=0.007)$ and quality of life $(-6.70 \pm 14.58, p=0.043)$. Over the intervention period, subjects taking $B$. lactis UABla-12 reported significant reductions as compared to placebo in IBS-SSS $(-104.5 \pm 96.08, p<0.001)$ with domain-specific scores related to abdominal pain severity $(-24.52 \pm 24.35, p<0.001)$, abdominal pain duration $(-20.95 \pm 23.91, p<0.001)$, abdominal distension $(-21.90 \pm 25.85, p=0.034)$ bowel habits $(-17.86 \pm 22.06, p=0.001)$ and quality of life $(-19.29$ $\pm 21.66, p=0.001)$. The $B$. lactis UABla-12 group also reported significant changes compared to placebo at the midpoint visit in IBS-SSS total score and domains related to abdominal pain severity, abdominal pain duration and bowel habits $(p<0.05)$. 
Table 1. Baseline demographics and clinical characteristics of the ITT population.

\begin{tabular}{|c|c|c|c|c|}
\hline & $\begin{array}{l}\text { Placebo } \\
(n=109)\end{array}$ & $\begin{array}{l}\text { L. acidophilus DDS-1 } \\
\quad(n=111)\end{array}$ & $\begin{array}{l}\text { B. lactis UABla-12 } \\
\quad(n=110)\end{array}$ & \multirow[t]{2}{*}{$p$ Value } \\
\hline & Mean (SD) or $n(\%)$ & Mean (SD) or $n(\%)$ & Mean (SD) or $n(\%)$ & \\
\hline Age (years) & $37.61(10.12)$ & $39.41(11.80)$ & $41.60(11.11)$ & $0.028 \ddagger$ \\
\hline Female gender, $n(\%)$ & $54(49.5)$ & $58(52.3)$ & $51(46.6)$ & $0.681^{+}$ \\
\hline BMI $\left(\mathrm{kg} / \mathrm{m}^{2}\right)$ & $24.10(4.06)$ & $24.09(4.34)$ & $23.78(3.98)$ & $0.811 \ddagger$ \\
\hline Systolic BP (kPa) & $15.89(1.13)$ & $15.96(1.08)$ & $16.03(1.11)$ & $0.660 \S$ \\
\hline Diastolic BP (kPa) & $10.09(0.94)$ & $10.33(0.86)$ & $10.25(1.00)$ & $0.116^{\S}$ \\
\hline Pulse Rate (bpm) & $78.23(8.63)$ & $76.96(7.69)$ & $78.18(8.10)$ & $0.426 \ddagger$ \\
\hline IBS duration (months) & $20.81(10.92)$ & $20.85(12.29)$ & $22.07(12.80)$ & $0.674 \ddagger$ \\
\hline GAD score & $3.88(1.81)$ & $4.04(1.62)$ & $4.16(1.64)$ & $0.378^{\S}$ \\
\hline PHQ-9 score & $5.38(3.15)$ & $5.61(3.05)$ & $5.32(2.96)$ & $0.749 \ddagger$ \\
\hline \multicolumn{5}{|l|}{ Alcohol consumption } \\
\hline None, $n(\%)$ & $96(88.1 \%)$ & $100(90.1 \%)$ & $97(88.2 \%)$ & \multirow{3}{*}{$0.983^{+}$} \\
\hline Occasional, $n(\%)$ & $3(2.8 \%)$ & $2(1.8 \%)$ & $3(2.7 \%)$ & \\
\hline Not available, $n(\%)$ & $10(9.2 \%)$ & $9(8.1 \%)$ & $10(9.1 \%)$ & \\
\hline
\end{tabular}

${ }^{\dagger}$ Between group comparison, Pearson Chi Square test; ${ }^{\ddagger}$ Between group comparison, one-way ANOVA; $§$ Between group comparison, Kruskal-Wallis test; BMI: body mass index; BP: blood pressure; IBS: irritable bowel syndrome; GAD: generalized anxiety disorder; PHQ: participant health questionnaire; SD: standard deviation.

Table 2. Abdominal pain severity scores over the intervention period.

\begin{tabular}{cccccc}
\hline & \multicolumn{3}{c}{ Mean (SD) } & & $p$ Value ${ }^{\dagger}$ \\
& $\begin{array}{c}\text { Placebo } \\
(\boldsymbol{n}=\mathbf{1 0 9})\end{array}$ & $\begin{array}{c}\text { L. acidophilus DDS-1 } \\
(\boldsymbol{n}=\mathbf{1 1 1})\end{array}$ & $\begin{array}{c}\text { B. lactis UABla-12 } \\
(\boldsymbol{n}=\mathbf{1 1 0})\end{array}$ & $\begin{array}{c}\text { DDS-1 vs. Placebo } \\
\text { UABla-12 vs. Placebo }\end{array}$ & $\begin{array}{c}\text { DDS-1 vs. } \\
\text { UABla-12 }\end{array}$ \\
\hline APS-NRS Score & & & & \\
Day 0 & $6.94(1.02)$ & $7.03(0.99)$ & $6.84(1.04)$ & 0.499 & 0.412 \\
Abs $\Delta$ (Day 21) & $-0.71(0.92)$ & $-1.24(1.10)$ & $-0.88(0.99)$ & $<0.001$ & 0.178 \\
Abs $\Delta$ (Day 42) & $-0.85(1.45)$ & $-2.59(2.07)$ & $-1.56(1.83)$ & 0.001 & 0.001 \\
\hline
\end{tabular}

${ }^{\dagger}$ Between group comparison, Mann-Whitney U test; APS-NRS: Abdominal Pain Severity - Numeric Rating Scale; Abs $\Delta$ : absolute change. 
Table 3. IBS symptom severity total and domain specific scores over the intervention period.

\begin{tabular}{|c|c|c|c|c|c|c|}
\hline & \multicolumn{3}{|c|}{ Mean (SD) } & \multicolumn{3}{|c|}{$p$ Value $^{\dagger}$} \\
\hline & $\begin{array}{l}\text { Placebo } \\
(n=109)\end{array}$ & $\begin{array}{l}\text { L. acidophilus DDS-1 } \\
\quad(n=111)\end{array}$ & $\begin{array}{l}\text { B. lactis UABla-12 } \\
\quad(n=110)\end{array}$ & $\begin{array}{l}\text { DDS-1 vs. } \\
\text { Placebo }\end{array}$ & $\begin{array}{l}\text { UABla-12 vs. } \\
\text { Placebo }\end{array}$ & $\begin{array}{l}\text { DDS-1 vs. } \\
\text { UABla-12 }\end{array}$ \\
\hline \multicolumn{7}{|l|}{ IBS-SSS Total Score } \\
\hline Day 0 & $298.07(55.68)$ & $310.90(52.47)$ & $305.45(48.82)$ & 0.108 & 0.324 & 0.504 \\
\hline $\operatorname{Abs} \Delta$ (Day 21) & $-30.09(57.76)$ & $-56.06(57.64)$ & $-50.00(63.72)$ & $<0.001$ & 0.020 & 0.185 \\
\hline $\operatorname{Abs} \Delta$ (Day 42) & $-55.70(86.42)$ & $-133.4(95.19)$ & $-104.5(96.08)$ & $<0.001$ & $<0.001$ & 0.039 \\
\hline \multicolumn{7}{|c|}{ Abdominal Pain Severity } \\
\hline Day 0 & $66.74(11.39)$ & $68.24(11.73)$ & $65.82(11.30)$ & 0.213 & 0.644 & 0.075 \\
\hline $\operatorname{Abs} \Delta$ (Day 21) & $-9.25(15.09)$ & $-15.50(15.23)$ & $-12.86(16.36)$ & 0.001 & 0.077 & 0.121 \\
\hline $\operatorname{Abs} \Delta($ Day 42) & $-13.41(20.17)$ & $-32.94(21.78)$ & $-24.52(24.35)$ & $<0.001$ & $<0.001$ & 0.009 \\
\hline \multicolumn{7}{|c|}{ Abdominal Pain Duration } \\
\hline Day 0 & $53.21(15.69)$ & $59.73(18.85)$ & $57.55(17.57)$ & 0.016 & 0.139 & 0.344 \\
\hline $\operatorname{Abs} \Delta$ (Day 21) & $-4.77(17.72)$ & $-13.49(21.79)$ & $-10.95(19.83)$ & 0.002 & 0.023 & 0.420 \\
\hline $\operatorname{Abs} \Delta$ (Day 42) & $-8.13(19.53)$ & $-28.44(24.80)$ & $-20.95(23.19)$ & $<0.001$ & $<0.001$ & 0.018 \\
\hline \multicolumn{7}{|c|}{ Abdominal Distension } \\
\hline Day 0 & $58.39(17.81)$ & $60.14(16.96)$ & $58.23(17.79)$ & 0.428 & 0.822 & 0.247 \\
\hline $\operatorname{Abs} \Delta$ (Day 21) & $-7.66(20.39)$ & $-11.47(16.26)$ & $-9.67(18.92)$ & 0.110 & 0.433 & 0.396 \\
\hline Abs $\Delta$ (Day 42) & $-15.47(23.81)$ & $-27.20(25.15)$ & $-21.90(25.85)$ & $<0.001$ & 0.034 & 0.098 \\
\hline \multicolumn{7}{|l|}{ Bowel Habits } \\
\hline Day 0 & $60.05(14.38)$ & $63.06(13.79)$ & $63.09(12.06)$ & 0.122 & 0.119 & 0.974 \\
\hline $\operatorname{Abs} \Delta$ (Day 21) & $-4.11(13.95)$ & $-8.90(13.82)$ & $-8.86(14.99)$ & 0.007 & 0.010 & 0.999 \\
\hline $\operatorname{Abs} \Delta$ (Day 42) & $-7.99(23.46)$ & $-22.89(22.46)$ & $-17.86(22.06)$ & $<0.001$ & 0.001 & 0.067 \\
\hline \multicolumn{7}{|c|}{ Effect on Quality of Life } \\
\hline Day 0 & $59.68(14.57)$ & $59.73(13.70)$ & $60.77(11.62)$ & 0.985 & 0.657 & 0.496 \\
\hline $\operatorname{Abs} \Delta$ (Day 21) & $-4.30(15.76)$ & $-6.70(14.58)$ & $-7.67(15.61)$ & 0.043 & 0.112 & 0.663 \\
\hline $\operatorname{Abs} \Delta$ (Day 42) & $-10.70(21.98)$ & $-21.88(22.27)$ & $-19.29(21.66)$ & $<0.001$ & 0.001 & 0.316 \\
\hline
\end{tabular}

${ }^{\dagger}$ Between group comparison, Mann-Whitney U test; IBS-SSS: Irritable Bowel Syndrome - Severity Scoring System. 


\subsection{Bowel Habits}

Participants in both probiotic groups showed a significant normalization in stool consistency over the intervention period when compared to placebo. As shown in Table 4, all three groups exhibited similar distributions in stool type at baseline. A significantly different distribution was observed in $L$. acidophilus DDS-1 ( $p=0.002)$ and B. lactis UABla-12 $(p=0.022)$ groups at day 42 as compared to placebo, with a higher percentage of subjects exhibiting normal stool type ( $83.8 \%$ or $75.5 \%$, respectively) and a correspondingly lower percentage of subjects with constipation and diarrhea stool types (Table 4).

Table 4. Stool consistency profile over the intervention period.

\begin{tabular}{ccccc}
\hline & & \multicolumn{3}{c}{ Subjects ( $\boldsymbol{n}, \mathbf{\%})$} \\
\hline Group & Stool type (BSS) & Day 0 & Day 21 & Day 42 \\
\hline \multirow{2}{*}{ Placebo } & Constipation (1-2) & $25(22.9)$ & $19(17.4)$ & $28(25.7)$ \\
& Normal (3-5) & $70(64.2)$ & $74(67.9)$ & $68(62.4)$ \\
& Diarrhea (6-7) & $14(12.8)$ & $14(12.8)$ & $11(10.1)$ \\
& Data not available & 0 & $2(1.8)$ & $2(1.8)$ \\
\hline \multirow{2}{*}{ L. acidophilus DDS-1 } & Constipation (1-2) & $24(21.6)$ & $14(12.6)$ & $9(8.1)$ \\
$(n=111)$ & Normal (3-5) & $63(56.8)$ & $82(73.9)$ & $93(83.8)$ \\
& Diarrhea (6-7) & $24(21.6)$ & $13(11.7)$ & $7(6.3)$ \\
& Data not available & 0 & $2(1.8)$ & $2(1.8)$ \\
\hline \multirow{2}{*}{ B. lactis UABla-12 } & Constipation (1-2) & $22(20.0)$ & $18(16.4)$ & $10(9.1)$ \\
$(n=110)$ & Normal (3-5) & $67(60.9)$ & $63(57.3)$ & $83(75.5)$ \\
& Diarrhea (6-7) & $21(19.1)$ & $24(21.8)$ & $12(10.9)$ \\
& Data not available & 0 & $5(4.5)$ & $5(4.5)$ \\
\hline \multirow{2}{*}{ Value ${ }^{\dagger}$} & DDS-1 vs. Placebo & 0.234 & 0.733 & 0.002 \\
& UABla-12 vs. Placebo & 0.438 & 0.444 & 0.022 \\
& DDS-1 vs. UABla-12 & 0.139 & 0.234 & 0.435 \\
\hline
\end{tabular}

${ }^{\dagger}$ Between group comparison, Pearson Chi Square test. BSS: Bristol Stool Scale.

Normalization of stool type was also observed via intragroup changes, as shown in Table 5, which tracked individual movements of stool types over the intervention period. The placebo group exhibited a roughly equal number of subjects transitioning from non-normal to normal vs. normal to non-normal stool types over the intervention period ( $p=1.000)$. In contrast, the L. acidophilus DDS-1 group showed a noticeable increase in participants transitioning to normal stool types at both day $21(p=0.006)$ and day $42(p<0.001)$, while the $B$. lactis UABla-12 showed a noticeable increase in participants transitioning to normal stool types at day $42(p=0.002)$.

Table 5. Intragroup change in stool type over the intervention period.

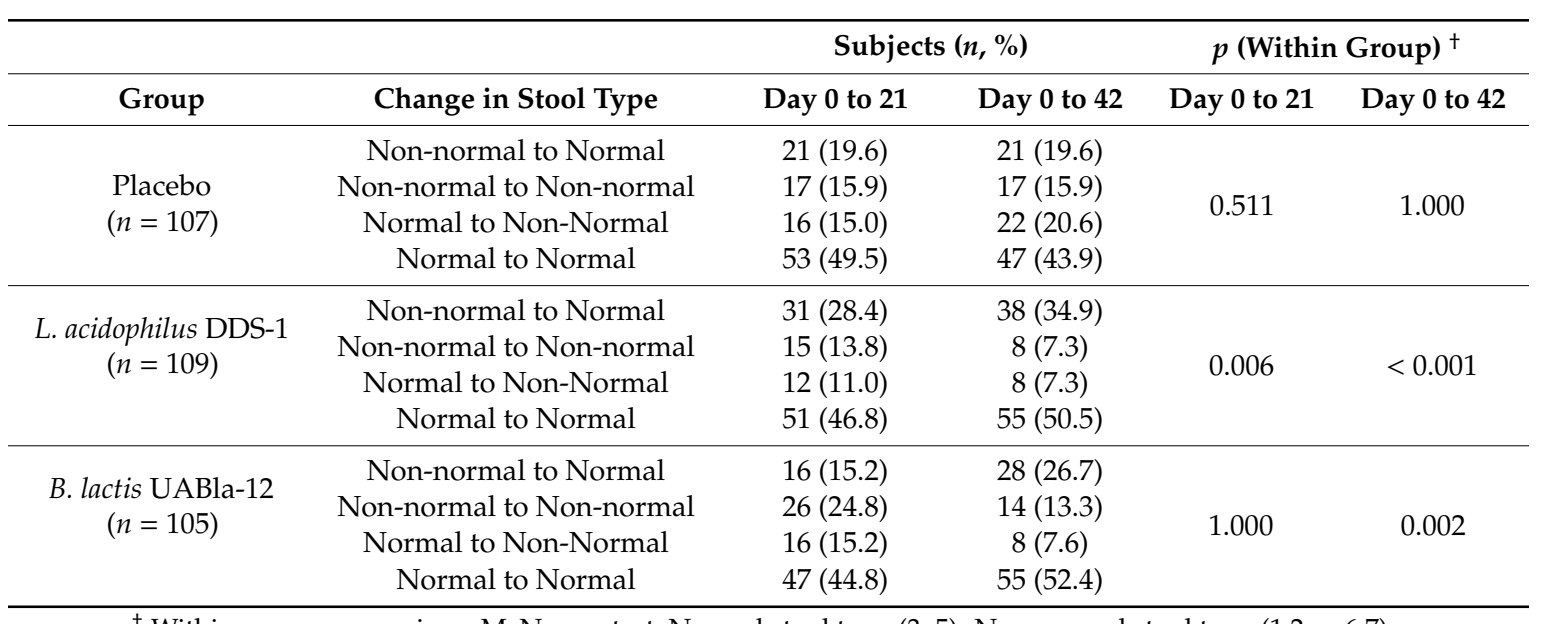

\footnotetext{
${ }^{+}$Within group comparison, McNemar test; Normal stool type (3-5). Non-normal stool type (1,2 or 6,7).
} 
A slight decrease in the daily number of stools was observed in both probiotic groups over the intervention period, however, no significant between-group differences were observed in stool frequency at any of the time points (Table S1).

\subsection{Quality of Life, Perceived Stress and Product Tolerability}

IBS-QoL scores were significantly improved in participants taking L. acidophilus DDS-1 as compared to placebo at the end of study visit $(p=0.016)$ (Table S2). PSS total scores were similarly improved at both day $21(p=0.002)$ and day $42(p=0.023)$ visits in subjects taking L. acidophilus DDS-1 as compared to placebo. Effects appeared to be more pronounced in sub-scores related to positive factors as compared to negative factors. In participants taking B. lactis UABla-12, despite intragroup improvement in IBS-QoL, no significant differences were observed when compared to placebo. Additionally, a significant effect was observed between B. lactis UABla-12 and placebo in PSS at day $21(p=0.030)$, however, no difference was observed at the end of study visit.

No differences were observed in study product tolerability between placebo and probiotic groups over the intervention period (Table S3). Similarly, no differences were observed in study product compliance, with mean compliance rates over the 42 day intervention period of $99 \%, 100 \%$ and $100 \%$ for the placebo, L. acidophilus DDS-1 and B. lactis UABla-12 groups, respectively (Table S3). Use of rescue medication was comparable and relatively rare, with approximately $90 \%$ of subjects not recording any use over the study period in all three groups $(p>0.05)$. Additionally, no significant changes were observed in safety variables in any group over the study period (Table S4).

\section{Discussion}

The present study was a randomized, double-blind, placebo-controlled trial to assess two probiotic strains, L. acidophilus DDS-1 and B. animalis subsp. lactis UABla-12, in adults with IBS. The study was unique in its simultaneous and independent assessment of a single strain Lactobacillus and Bifidobacterium in a well-powered three-arm trial of IBS patients across 12 clinical sites. A dose of NLT 1 $\times 10^{10} \mathrm{CFU}$ daily was administered as it is supported by prior gastrointestinal focused randomized controlled trials on the strains $[12,13]$ and is a midpoint dosage in meta-analyses of probiotic studies for IBS [9]. Enrolled participants were primarily young to middle-aged adults, with a mixed gender allocation and a normal to overweight BMI, representing a typical cross-section of IBS in India and elsewhere [27]. The lack of an obvious female predominance among IBS subjects in this study is in agreement with other Asian reports, and in contrast to a 2-3-fold greater prevalence among females in Western populations [27,28]. There is an unmet need for alleviation of IBS symptoms in Indian clinical practices, particularly in urban areas. Chronic gut infections, gut microbiota dysbiosis, and small intestinal bacterial overgrowth are suggested as contributing factors in South Asian populations [28].

Both strains achieved the primary outcome, reducing abdominal pain severity over the intervention period, when compared to placebo. The US Food and Drug Administration (FDA) has recommended the use of the 11-point numeric rating scale for abdominal pain severity, assessed here as primary outcome, for pain in IBS [29]. Further, the APS-NRS is both well validated and can be readily interpreted with a clinically important difference in IBS [21].

An intervention period of six weeks was assessed for the primary outcome, as it is consistent with the four- to eight-week probiotic IBS study durations widely reported in the literature [9]. Placebo response is significant in IBS studies and has been demonstrated to be highest in trials with a one- to four-week intervention period [30]. An initial increase in placebo response has been reported before stabilizing over two to five weeks and decreasing after 12 weeks [31,32]. For this reason, it has been suggested to include an intervention period of at least five weeks, and preferably longer, to avoid a potential unstable placebo group response [33]. Alternatively, other reviews report a higher placebo response associated with longer treatment duration and a greater number of study visits [34]. While the current intervention period was relatively short at six weeks, it incorporated design considerations [33], 
including a placebo run-in period, exclusion criteria regarding placebo responders, relatively few study visits and a large sample size to ensure adequate power.

FDA guidance defines a responder as a decrease in weekly average abdominal pain of at least $30 \%$ compared to baseline [29]. Clinically significant responders in the current study, considering the entire ITT population including dropouts, were $52.3 \%$ and $28.2 \%$ in the L. acidophilus DDS- 1 and $B$. lactis UABla-12 groups respectively, both of which were significantly higher than placebo $(15.6 \%)$. It is of interest that both probiotic strains, most notably L. acidophilus DDS-1, showed a significant group response as well an individual response to intervention. The meaningfulness of individual responses to treatment may, in some cases, be of more interest than group responses [35], and thus both are reported here. The high responder rates observed herein may also be applicable more generally. Episodes of abdominal pain occur in healthy people as well as individuals with IBS with the difference being the frequency and severity of symptoms [36]. Therefore, according to the European Food Safety Authority, IBS patients are generally an appropriate study group to substantiate claims related to gastrointestinal discomfort in the general population [36].

Participants taking L. acidophilus DDS-1 and B. lactis UABla-12 exhibited normalization of stool type in concert with improved outcomes in abdominal pain severity. Previously, a probiotic blend including the two strains studied here helped modulate bowel habits in functional constipation [12]. In the current study, a reduction in both constipation and diarrhea related stool types and a corresponding increase in normal stool types was observed, most noticeably with L. acidophilus DDS-1. A significant change in stool type was observed despite no significant alteration in stool frequency. Basal levels of stool frequency tend to be relatively higher in India [27]. Further, a patient's description of constipation or diarrhea based on stool frequency alone can often be erroneous [37]. It has therefore been suggested to include stool type for a better assessment of bowel patterns in Indian populations [37].

Both probiotic strains helped improve total symptomology as per the validated questionnaire, with changes that could be classified as clinically significant. It has been reported that a minimally important clinical difference for the IBS-SSS is 95 points and thus patients exceeding this change could be classified as a responder [21]. Domains related to abdominal pain severity and duration, bloating, bowel habits and quality of life were also improved in probiotic groups as compared to placebo.

Multiple reports have linked IBS pathogenesis to dysbiosis of the microbiota [38,39], including low levels of Lactobacillus and Bifidobacterium [40], which could otherwise help inhibit binding of pathogens and reinforce defenses of the mucosal barrier. The Rome Team Working Group has supported the concept that the intestinal microbiota is perturbed in IBS [41]. There is evidence of an activation of the intestinal immune system in IBS resulting in inflammation, including augmented mucosal intraepithelial lymphocytes and mast cells [42,43].

The gut microbiota also plays an important role in the balance between immunosuppression and inflammation, involving Toll-like receptor signaling pathways [39]. L. acidophilus DDS-1 was previously shown to help modulate the fecal and mucosal microbiota in young and aging mice, while downregulating the production of inflammatory cytokines in serum and colonic explants $[17,18]$. Additionally, L. acidophilus DDS-1, in combination with B. lactis UABla-12, displayed improved symptomology associated with atopic dermatitis in a randomized controlled trial, with modulation of blood lymphocyte subsets (i.e., CD4, CD8 and CD25) suggesting an immune regulatory role [15].

In addition to the above, the microbiota can modulate visceral afferent pathways by effecting enterocytes, enteroendocrine cells, and the neurons themselves [44]. L. acidophilus has been shown to normalize visceral pain responses via induced expression of opioid and cannabinoid receptors in the gut [45]. Similarly, the species has been shown to modulate $\mu$-opioid receptor expression and activity, which has been suggested as a mechanism for the reduction of bloating in adults with functional abdominal pain [46,47]. More recently, Lactobacillus was shown to upregulate expression of both serotonin transporter and intestinal serotonin levels [48], which further suggests a potential gut-brain role. In addition to the regulation of pain, mental stress was decreased with L. acidophilus DDS- 1 in the 
current study. As IBS is considered a stress sensitive disorder [49], improved stress levels are possibly linked to a more normalized immune response, intestinal motility or barrier function.

Limitations of this study include the absence of microbial profiling or biomarker analysis, in part due to the large enrollment across many clinical sites. Further, while dietary guidelines were provided to subjects throughout the study, a dietary analysis was not performed. A recent study demonstrated a unique composition of the Indian gut microbiome, including diverging profiles of cohorts from North-Central and South India, who were primarily consuming plant-based and omnivorous diets, respectively [50]. Based on available evidence for the current study, omnivorous diets outnumbered plant-based diets in all three groups, with an overall ratio of 2.1 to 1 . Correlation of the microbial community with diet and other metadata in the context of responders and non-responders would have been of interest to explore given the results of the current study.

Nevertheless, the study was well-powered to assess its primary and secondary outcome, it took into account integrated study design considerations for IBS [33], including a placebo run-in period and alignment of the study inclusion with the study outcomes, and outcomes were assessed over numerous clinical sites. In conclusion, abdominal pain severity was significantly improved in subjects receiving L. acidophilus DDS-1 or B. lactis UABla-12, both in terms of group response and individual response to intervention. Further, both probiotic strains showed a clinically significant alleviation of symptom severity with a corresponding normalization of bowel habits in males and females with IBS.

Supplementary Materials: The following are available online at http://www.mdpi.com/2072-6643/12/2/363/s1, Table S1: Daily stool frequency over intervention period Table S2: IBS quality of life and perceived stress scores over intervention period; Table S3: Study product tolerability and compliance over intervention period; Table S4: Safety variables over intervention period.

Author Contributions: C.J.M. and G.J.L. designed the study and prepared the manuscript. S.S. directed the study, data analysis and reporting. All authors have read and agreed to the published version of the manuscript.

Funding: This research was funded by UAS Laboratories LLC.

Acknowledgments: We thank the medical investigators for overseeing the conduct of the study. We also thank the study participants, monitors, study nurses, project and quality assurance managers, data analysts and statisticians who enabled this study. We are thankful to S. Basirico for overseeing the production and quality control of the study products.

Conflicts of Interest: Christopher J. Martoni and Gregory Leyer are employees of UAS Laboratories but were not involved in the study conduct, data management or statistical analysis.

\section{References}

1. Holtmann, G.J.; Ford, A.C.; Talley, N.J. Pathophysiology of irritable bowel syndrome. Lancet Gastroenterol. Hepatol. 2016, 1, 133-146. [CrossRef]

2. Barbara, G.; Feinle-Bisset, C.; Ghoshal, U.C.; Santos, J.; Vanner, S.J.; Vergnolle, N.; Zoetendal, E.G.; Quigley, E.M. The intestinal microenvironment and functional gastrointestinal disorders. Gastroenterology 2016, 150, 1305-1318. [CrossRef]

3. Dale, H.F.; Rasmussen, S.H.; Asiller, Ö.Ö.; Lied, G.A. Probiotics in Irritable Bowel Syndrome: An Up-to-Date Systematic Review. Nutrients 2019, 11, 2048. [CrossRef]

4. Lacy, B.; Patel, N. Rome Criteria and a Diagnostic Approach to Irritable Bowel Syndrome. J. Clin. Med. 2017, 6, 99. [CrossRef] [PubMed]

5. Ford, A.C.; Moayyedi, P.; Chey, W.D.; Harris, L.A.; Lacy, B.E.; Saito, Y.A.; Quigley, E.M.M. ACG Task Force on Management of Irritable Bowel Syndrome American College of Gastroenterology Monograph on Management of Irritable Bowel Syndrome. Am. J. Gastroenterol. 2018, 113, 1-18. [CrossRef] [PubMed]

6. Barbara, G.; Cremon, C.; Azpiroz, F. Probiotics in irritable bowel syndrome: Where are we? Neurogastroenterol. Motil. 2018, 30, 1-6. [CrossRef] [PubMed]

7. Ford, A.C.; Harris, L.A.; Lacy, B.E.; Quigley, E.M.M.; Moayyedi, P. Systematic review with meta-analysis: The efficacy of prebiotics, probiotics, synbiotics and antibiotics in irritable bowel syndrome. Aliment. Pharmacol. Ther. 2018, 48, 1044-1060. [CrossRef] [PubMed] 
8. Didari, T.; Mozaffari, S.; Nikfar, S.; Abdollahi, M. Effectiveness of probiotics in irritable bowel syndrome: Updated systematic review with meta-analysis. World J. Gastroenterol. 2015, 21, 3072-3084. [CrossRef]

9. Zhang, Y.; Li, L.; Guo, C.; Mu, D.; Feng, B.; Zuo, X.; Li, Y. Effects of probiotic type, dose and treatment duration on irritable bowel syndrome diagnosed by Rome III criteria: A meta-analysis. BMC Gastroenterol. 2016, 16, 1-11. [CrossRef]

10. Hill, C.; Guarner, F.; Reid, G.; Gibson, G.R.; Merenstein, D.J.; Pot, B.; Morelli, L.; Canani, R.B.; Flint, H.J.; Salminen, S.; et al. Expert consensus document: The International Scientific Association for Probiotics and Prebiotics consensus statement on the scope and appropriate use of the term probiotic. Nat. Rev. Gastroenterol. Hepatol. 2014, 11, 506-514. [CrossRef]

11. Ford, A.C.; Moayyedi, P.; Lacy, B.E.; Lembo, A.J.; Saito, Y.A.; Schiller, L.R.; Soffer, E.E.; Spiegel, B.M.R.; Quigley, E.M.M. American college of gastroenterology monograph on the management of irritable bowel syndrome and chronic idiopathic constipation. Am. J. Gastroenterol. 2014, 109, S2-S26. [CrossRef]

12. Martoni, C.J.; Evans, M.; Chow, C.T.; Chan, L.S.; Leyer, G. Impact of a probiotic product on bowel habits and microbial profile in participants with functional constipation: A randomized controlled trial. J. Dig. Dis. 2019, 20, 435-446. [CrossRef] [PubMed]

13. Pakdaman, M.N.; Udani, J.K.; Molina, J.P.; Shahani, M. The effects of the DDS-1 strain of lactobacillus on symptomatic relief for lactose intolerance - A randomized, double-blind, placebo-controlled, crossover clinical trial. Nutr. J. 2016, 15. [CrossRef] [PubMed]

14. Gerasimov, S.V.; Ivantsiv, V.A.; Bobryk, L.M.; Tsitsura, O.O.; Dedyshin, L.P.; Guta, N.V.; Yandyo, B.V. Role of short-term use of L. acidophilus DDS-1 and B. lactis UABLA-12 in acute respiratory infections in children: A randomized controlled trial. Eur. J. Clin. Nutr. 2016, 70, 463-469. [CrossRef] [PubMed]

15. Gerasimov, S.V.; Vasjuta, V.V.; Myhovych, O.O.; Bondarchuk, L.I. Probiotic supplement reduces Atopic Dermatitis in preschool children: A randomized, double-blind, placebo-controlled, clinical trial. Am. J. Clin. Dermatol. 2010, 11, 351-361. [CrossRef]

16. Vemuri, R.; Shinde, T.; Shastri, M.D.; Perera, A.P.; Tristram, S.; Martoni, C.J.; Gundamaraju, R.; Ahuja, K.D.K.; Ball, M.; Eri, R. A human origin strain Lactobacillus acidophilus DDS-1 exhibits superior in vitro probiotic efficacy in comparison to plant or dairy origin probiotics. Int. J. Med. Sci. 2018, 15, 840-848. [CrossRef] [PubMed]

17. Vemuri, R.; Shinde, T.; Gundamaraju, R.; Gondalia, S.V.; Karpe, A.V.; Beale, D.J.; Martoni, C.J.; Eri, R. Lactobacillus acidophilus DDS-1 modulates the gut microbiota and improves metabolic profiles in aging mice. Nutrients 2018, 10, 1255. [CrossRef] [PubMed]

18. Vemuri, R.; Gundamaraju, R.; Shinde, T.; Perera, A.P.; Basheer, W.; Southam, B.; Gondalia, S.V.; Karpe, A.V.; Beale, D.J.; Tristram, S.; et al. Lactobacillus acidophilus DDS-1 modulates intestinal-specific microbiota, short-chain fatty acid and immunological profiles in aging mice. Nutrients 2019, 11, 1297. [CrossRef] [PubMed]

19. Nagala, R.; Routray, C. Clinical Case Study-Multispecies probiotic supplement minimizes symptoms of irritable bowel syndrome. US Gastroenterol. Hepatol. Rev. 2011, 7, 1-2.

20. Spiegel, B.M.R.; Bolus, R.; Harris, L.A.; Lucak, S.; Chey, W.D.; Sayuk, G.; Esrailian, E.; Lembo, A.; Karsan, H.; Tillisch, K.; et al. Characterizing abdominal pain in IBS: Guidance for study inclusion criteria, outcome measurement and clinical practice. Aliment. Pharmacol. Ther. 2010, 32, 1192-1202. [CrossRef] [PubMed]

21. Spiegel, B.; Bolus, R.; Harris, L.A.; Lucak, S.; Naliboff, B.; Esrailian, E.; Chey, W.D.; Lembo, A.; Karsan, H.; Tillisch, K.; et al. Measuring IBS patient reported outcomes with an abdominal pain numeric rating scale: Results from the proof cohort. Aliment. Pharmacol. Ther. 2009, 30, 1159-1170. [CrossRef] [PubMed]

22. Spiegel, B.M.R.; Bolus, R.; Agarwal, N.; Sayuk, G.; Harris, L.A.; Lucak, S.; Esrailian, E.; Chey, W.D.; Lembo, A.; Karsan, H.; et al. Measuring symptoms in the irritable bowel syndrome: Development of a framework for clinical trials. Aliment. Pharmacol. Ther. 2010, 32, 1275-1291. [CrossRef] [PubMed]

23. Francis, C.Y.; Morris, J.; Whorwell, P.J. The irritable bowel severity scoring system: A simple method of monitoring irritable bowel syndrome and its progress. Aliment. Pharmacol. Ther. 1997, 11, 395-402. [CrossRef] [PubMed]

24. Blake, M.R.; Raker, J.M.; Whelan, K. Validity and reliability of the Bristol Stool Form Scale in healthy adults and patients with diarrhoea-predominant irritable bowel syndrome. Aliment. Pharmacol. Ther. 2016, 44, 693-703. [CrossRef] [PubMed] 
25. Patrick, D.L.; Drossman, D.A.; Frederick, I.O.; Dicesare, J.; Puder, K.L. Quality of life in persons with irritable bowel syndrome: Development and validation of a new measure. Dig. Dis. Sci. 1998, 43, 400-411. [CrossRef] [PubMed]

26. Cohen, S.; Williamson, G. Perceived stress in a probability sample of the United States. Soc. Psychol. Heal. 1988, 31-67.

27. Ghoshal, U.C.; Abraham, P.; Bhatt, C.; Choudhuri, G.; Bhatia, S.J.; Shenoy, K.T.; Banka, N.H.; Bose, K.; Bohidar, N.P.; Chakravartty, K.; et al. Epidemiological and clinical profile of irritable bowel syndrome in India: Report of the Indian Society of Gastroenterology Task Force. Indian J. Gastroenterol. 2008, 27, 22-28.

28. Masudur Rahman, M.; Mahadeva, S.; Ghoshal, U.C. Epidemiological and clinical perspectives on irritable bowel syndrome in India, Bangladesh and Malaysia: A review. World J. Gastroenterol. 2017, 23, 6788-6801. [CrossRef]

29. Food and Drug Administration. Guidance for Industry: Irritable Bowel Syndrome-Clinical Evaluation of Drugs for Treatment; Docket No. FDA-2012-D-0146; Food and Drug Administration: Silver Spring, MD, USA, 2012; pp. 32124-32125.

30. Ford, A.C.; Moayyedi, P. Meta-analysis: Factors affecting placebo response rate in the irritable bowel syndrome. Aliment. Pharmacol. Ther. 2010, 32, 144-158. [CrossRef] [PubMed]

31. Spiller, R.C. Problems and challenges in the design of irritable bowel syndrome clinical trials: Experience from published trials. Am. J. Med. 1999, 107, 91S-97S. [CrossRef]

32. Mangel, A.W. Study design issues in irritable bowel syndrome. Aliment. Pharmacol. Ther. 2004, 19, 141-142. [CrossRef]

33. Miller, L.E. Study design considerations for irritable bowel syndrome clinical trials. Ann. Gastroenterol. $Q$. Publ. Hell. Soc. Gastroenterol. 2014, 27, 338-345.

34. Dorn, S.D.; Kaptchuk, T.J.; Park, J.B.; Nguyen, L.T.; Canenguez, K.; Nam, B.H.; Woods, K.B.; Conboy, L.A.; Stason, W.B.; Lembo, A.J. A meta-analysis of the placebo response in complementary and alternative medicine trials of irritable bowel syndrome. Neurogastroenterol. Motil. 2007, 19, 630-637. [CrossRef] [PubMed]

35. Snapinn, S.M.; Jiang, Q. Responder analyses and the assessment of a clinically relevant treatment effect. Trials 2007, 8, 31. [CrossRef]

36. European Food Safety Authority (EFSA). Guidance on the scientific requirements for health claims related to gut and immune function. EFSA J. 2011, 9, 1984. [CrossRef]

37. Upadhyay, R.; Singh, A. Irritable Bowel Syndrome: The Indian Scenario. Med. Updat. 2013, 255-258.

38. Chong, P.P.; Chin, V.K.; Looi, C.Y.; Wong, W.F.; Madhavan, P.; Yong, V.C. The microbiome and irritable bowel syndrome - A review on the pathophysiology, current research and future therapy. Front. Microbiol. 2019, 10, 1-23.

39. Distrutti, E.; Monaldi, L.; Ricci, P.; Fiorucci, S. Gut microbiota role in irritable bowel syndrome: New therapeutic strategies. World J. Gastroenterol. 2016, 22, 2219-2241. [CrossRef]

40. Bellini, M.; Gambaccini, D.; Stasi, C.; Urbano, M.T.; Marchi, S.; Usai-Satta, P. Irritable bowel syndrome: A disease still searching for pathogenesis, diagnosis and therapy. World J. Gastroenterol. 2014, 20, 8807-8820.

41. Simren, M.; Palsson, O.S.; Whitehead, W.E. Update on Rome IV Criteria for Colorectal Disorders: Implications for Clinical Practice. Curr. Gastroenterol. Rep. 2017, 19. [CrossRef] [PubMed]

42. Chadwick, V.S.; Chen, W.; Shu, D.; Paulus, B.; Bethwaite, P.; Tie, A.; Wilson, I. Activation of the mucosal immune system in irritable bowel syndrome. Gastroenterology 2002, 122, 1778-1783. [CrossRef]

43. Guilarte, M.; Santos, J.; De Torres, I.; Alonso, C.; Vicario, M.; Ramos, L.; Martinez, C.; Casellas, F.; Saperas, E.; Malagelada, J.R. Diarrhoea-predominant IBS patients show mast cell activation and hyperplasia in the jejunum. Gut 2007, 56, 203-209. [CrossRef]

44. Lomax, A.E.; Pradhananga, S.; Sessenwein, J.L.; O’Malley, D. Bacterial modulation of visceral sensation: Mediators and mechanisms. Am. J. Physiol. Liver Physiol. 2019, 317, G363-G372. [CrossRef] [PubMed]

45. Rousseaux, C.; Thuru, X.; Gelot, A.; Barnich, N.; Neut, C.; Dubuquoy, L.; Dubuquoy, C.; Merour, E.; Geboes, K.; Chamaillard, M.; et al. Lactobacillus acidophilus modulates intestinal pain and induces opioid and cannabinoid receptors. Nat. Med. 2007, 13, 35-37. [CrossRef] [PubMed]

46. Ringel-Kulka, T.; Palsson, O.S.; Maier, D.; Carroll, I.; Galanko, J.A.; Leyer, G.; Ringel, Y. Probiotic bacteria Lactobacillus acidophilus NCFM and Bifidobacterium lactis Bi-07 versus placebo for the symptoms of bloating in patients with functional bowel disorders: A double-blind study. J. Clin. Gastroenterol. 2011, 45, 518-525. [CrossRef] 
47. Ringel-Kulka, T.; Goldsmith, J.R.; Carroll, I.M.; Barros, S.P.; Palsson, O.; Jobin, C.; Ringel, Y. Lactobacillus acidophilus NCFM affects colonic mucosal opioid receptor expression in patients with functional abdominal pain - A randomised clinical study. Aliment. Pharmacol. Ther. 2014, 40, 200-207. [CrossRef]

48. Ranuh, R.; Athiyyah, A.F.; Darma, A.; Risky, V.P.; Riawan, W.; Surono, I.S.; Sudarmo, S.M. Effect of the probiotic lactobacillus plantarum is- 10506 on bdnf and 5 ht stimulation: Role of intestinal microbiota on the gut-brain axis. Iran. J. Microbiol. 2019, 11, 145-150. [CrossRef]

49. Qin, H.Y.; Cheng, C.W.; Tang, X.D.; Bian, Z.X. Impact of psychological stress on irritable bowel syndrome. World J. Gastroenterol. 2014, 20, 14126-14131. [CrossRef]

50. Dhakan, D.B.; Maji, A.; Sharma, A.K.; Saxena, R.; Pulikkan, J.; Grace, T.; Gomez, A.; Scaria, J.; Amato, K.R.; Sharma, V.K. The unique composition of Indian gut microbiome, gene catalogue, and associated fecal metabolome deciphered using multi-omics approaches. Gigascience 2019, 8, 1-20. [CrossRef] [PubMed]

C 2020 by the authors. Licensee MDPI, Basel, Switzerland. This article is an open access article distributed under the terms and conditions of the Creative Commons Attribution (CC BY) license (http://creativecommons.org/licenses/by/4.0/). 